
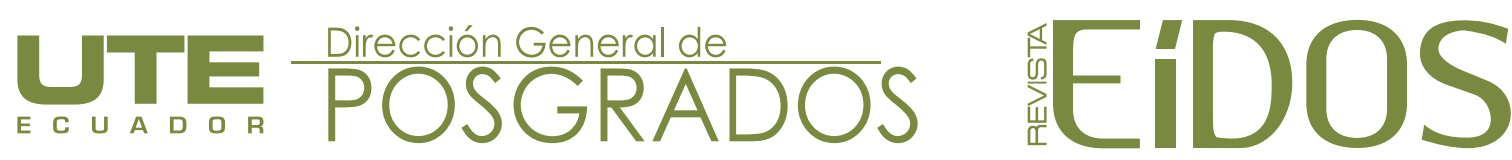

\title{
DENTICIÓN MIXTA: ESTUDIO COMPARATIVO DE ANÁLISIS DE ESPACIOS CON PRESENCIA O AUSENCIA DE MOLARES TEMPORALES EN NIÑOS DE 7 A 9 AÑOS
}

\author{
${ }^{1}$ María Paulina Sempértegui Sandoval, ${ }^{2 B}$ Byron Marcelo Villarreal Ortega
}

1Universidad Tecnológica Equinoccial, Quito, Ecuador, mapausesa@gmail.com

2Universidad Tecnológica Equinoccial, Quito - Ecuador, bvillareal@ute.edu.ec

Recepción / Received: 26, 08, 2014

Aceptación / Accepted: 03, 12, 2014

Publicado / Published: 12, 12, 2014

\section{RESUMEN:}

En etapa de dentición mixta es frecuente encontrar pérdida temprana de piezas temporales, junto con alteraciones estéticas y funcionales, que ocasionan migraciones o rotaciones de piezas vecinas y dan como resultado una pérdida del espacio disponible en las arcadas, lo que se torna desfavorable para la erupción de las piezas permanentes, que no tendrán el espacio necesario para su alineación normal. El objetivo de la presente investigación fue el de determinar la discrepancia dentaria entre espacio disponible y requerido de arcadas dentarias con presencia o ausencia de molares temporales, según el método de Moyers. Aquellos pacientes que presentaron todos sus molares temporales, tienen una mayor longitud del arco (superior e inferior), en comparación a aquellos con ausencia de molares temporales, en donde Sig. $=0.002<0.05$ para el arco superior y Sig. $=0.000<0.05$ para el arco inferior. La ausencia de por lo menos un molar temporal, produce una disminución del espacio, lo que conlleva a alteraciones funcionales y estéticas.

\section{PALABRAS CLAVE:}

Dentición mixta, discrepancias dentarias, espacio disponible, espacio requerido.

\begin{abstract}
:
It is common to find in mixed dentition stages, early loss of deciduous teeth along with consequent aesthetics and functional changes. There can be migrations or rotations of the neighboring teeth, causing a loss of available space in the arcades, being unfavorable to the eruption of the permanent teeth, which will not have the space to its normal alignment. The goal of this study was to find if dental discrepancies between available space and required space of dental arches separate in two groups, one with absence of at least one temporary molar, and one with presence of all deciduous teeth, according to the Moyer's method. We found that those patients who had all their molars in arches, the arc length was bigger (upper and lower), compared to those with absence of at least one molar; where Sig $=0.002<0.05$ is for the upper arch and Sig $=0.000<0.05$ is for the lower arch. The presence of all deciduous teeth is favorable for the permanent teeth to erupt into the dental arches, however, the absence of at least one molar, causes a decrease in space length, which leads to functional and aesthetic alterations.
\end{abstract}

\section{KEY WORDS:}

Mixed dentition, dental discrepancies, available space, required space 


\section{INTRODUCCIÓN}

La dentición mixta es una de las etapas del desarrollo normal del aparato estomatológico del ser humano. Esta inicia con la erupción de los incisivos centrales inferiores permanentes, junto con la de los primeros molares superiores e inferiores permanentes y culmina cuando la última pieza temporal ha terminado con su proceso de exfoliación.1 Cada una de las piezas dentales temporales tiene su posición y su cronología de erupción, de manera que actúan como mantenedores de espacio y guías para la erupción de los dientes permanentes, además de otras funciones como la fonación y la masticación. (Hubertus, J.M. \& Van, W. \& Paul, W. S. 2002).

Es frecuente encontrar pacientes en etapa de dentición mixta con ausencia prematura de molares temporales, lo que conlleva a la pérdida de espacio en la arcada dental, provocando apiñamientos dentales futuros, lo cual constituye una de las alteraciones más comunes en el paciente adulto. (Gutierrez Pulido, L. 2006), así como otro tipo de anomalías oclusales, como lo menciona Northway (1984) en un estudio realizado sobre los efectos significativos y duraderos de la pérdida temprana de piezas temporales. (Ramírez M. Perona G. 2011)

Para predecir si una persona tendrá o no suficiente espacio en las arcadas dentales, es necesario evaluar los espacios requeridos y disponibles de las mismas. Espacio disponible es aquel con el que contamos para la erupción de los caninos y primero y segundo premolar. Por otro lado, espacio requerido es el necesario para que se dé una erupción normal de las mismas piezas; por último, la discrepancia dentoalveolar es la diferencia que existe entre espacio disponible y espacio requerido, la cual puede resultar positiva, en caso de que haya más espacio del necesario; y negativa, en caso de que que exista espacio insuficiente o nula, cuando el espacio disponible y el requerido son los mismos. (Velásquez D. 2011).

El presente estudio tiene como objetivo analizar los espacios de arcadas dentarias en dentición mixta de dos grupos: aquellos con presencia de molares temporales y aquellos con ausencia de por lo menos uno de ellos, y comparar los resultados, para determinar si estos son o no estadísticamente significativos.

Para esto se aplicó, como herramienta, la tabla de Moyers (Gutiérrez, 2006) para predecir el tamaño mesio distal de caninos y premolares permanentes no erupcionados, a partir de una sumatoria individual de los cuatro incisivos inferiores deciduos. De esta manera se podría determinar si una persona tendría o no el espacio necesario para la erupción correcta de sus piezas permanentes y, de cierta manera, se intenta crear conciencia en las personas sobre el cuidado adecuado de las piezas dentales temporales, y cómo la ausencia de por lo menos una pieza, podría traer repercusiones a futuro.

Con estos antecedentes podemos determinar la importancia del Análisis de Moyers como un auxiliar de diagnóstico de las malas oclusiones, dándole una relevancia importante en las áreas de conocimiento de la odontología, ya que con estos análisis de espacio a edad temprana, podemos evitar el uso indiscriminado de expansiones y extracciones a futuro.

\section{POBLACIÓN ESTUDIO}

La población y muestra elegida en esta investigación fueron niños de 7 a 9 años de edad de una escuela fiscal de la ciudad de Quito. Al ser un estudio de análisis de espacios en dentición mixta, esta población etaria es la ideal, ya que presentan piezas temporales y piezas definitivas al mismo tiempo.

\section{MATERIALES Y MÉTODOS}

Para iniciar con la investigación se tomaron impresiones dentales a la población con alginato. La técnica de mezcla fue realizada cuidando que las proporciones de agua y polvo fueran las determinadas por el fabricante. La mezcla de este material se realizó en tazas de plástico con una espátula, hasta conseguir una pasta homogénea sin grumos ni burbujas. Se procedió a vaciar los modelos con yeso de ortodoncia inmediatamente después de haber obtenido las muestras, para no alterar la estabilidad dimensional de la impresión. Además, se colocaron los modelos en sócalos y se trimaron de acuerdo a las medidas de ortodoncia. Una vez retirados los modelos de estudio, se realizó la medición de los espacios de las arcadas.

Para la medición de los segmentos de cada modelo se utilizó un calibrador pie de rey metálico, con el que se midió en milímetros el diámetro mesio distal de los cuatro incisivos inferiores y cada valor fue registrado en la tabla de recolección de información, un ejemplo de la cual consta en la Tabla 1.

Tabla 1. Tabla de recolección de la información.

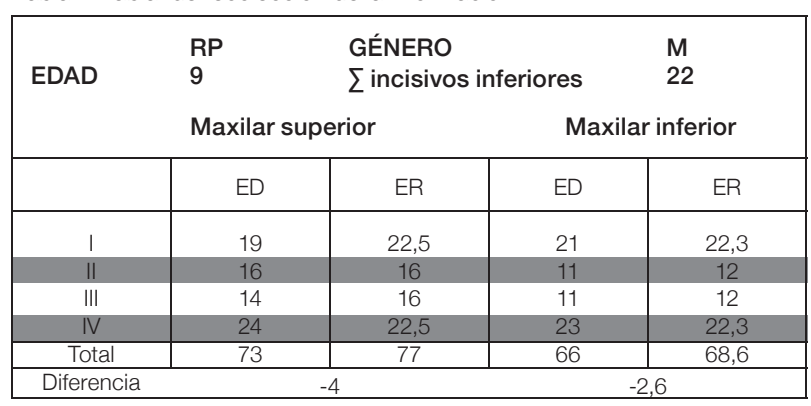


Se tomaron dos medidas:

Espacio disponible: para los cual se dividió a los modelos en 4 segmentos (Figura 1).

- Segmento 1: Distancia de la superficie mesial de primer molar permanente derecho hasta superficie distal de incisivo lateral derecho.

- Segmento 2: Distancia de la superficie distal de incisivo lateral derecho hasta la línea media.

- Segmento 3: Distancia de la superficie distal de incisivo lateral izquierdo hasta la línea media.

- Segmento 4: Distancia de la superficie mesial de primer molar permanente izquierdo hasta la superficie distal del incisivo lateral izquierdo.

Para esto se determinó la cantidad de espacio necesario a fin de realizar la alineación de los incisivos inferiores y se marcó en la cresta alveolar de cada lado, donde quedaría la cara distal de los incisivos laterales.

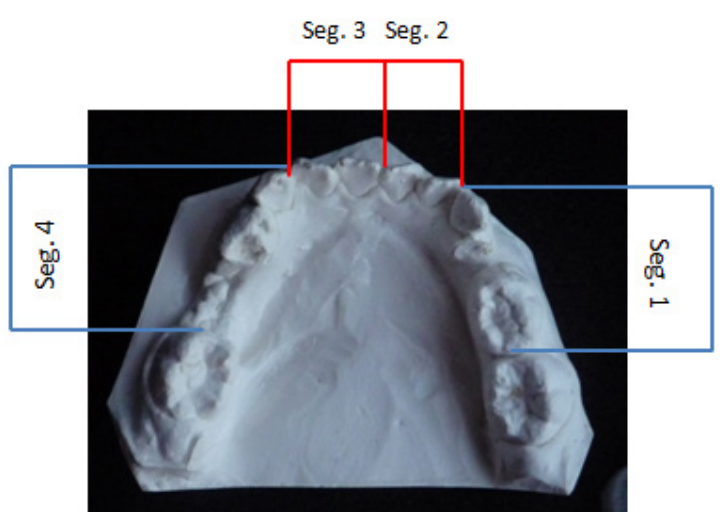

Figura № 1. División del modelo por segmentos.

Espacio requerido: se procedió a medir el ancho mesio distal de cada uno de los incisivos inferiores y a registrar en la TRI en los segmentos 2 y 3 , respectivamente. Con la sumatoria de estos valores, se utilizó la tabla de probabilidades de Moyers (Gutiérrez, 2006).

En la tabla se ubicó el valor correspondiente a la sumatoria de los cuatro incisivos, y se eligió el nivel de percentil al $75 \%$, y el valor que se obtuvo, se registró en los segmentos 1 y 4 . De este modo, se obtuvo la predicción de los anchos de caninos y premolares inferiores.

El procedimiento para el arco superior es similar al de inferior, con dos diferencias significativas:

- Existe una tabla de probabilidades diferente para la arcada superior.

- La predicción del tamaño de caninos y premolares superiores se realiza también en base a la sumatoria de los anchos mesio distales de los incisivos inferiores permanentes.
Se realizó la sumatoria total, tanto de espacio disponible, como de espacio requerido, de maxilar superior y de maxilar inferior.

Una vez obtenidos estos valores, se restaron los tamaños calculados. (Espacio Disponible - Espacio Requerido). Así se obtuvieron los resultados que se clasifican en:

a. Discrepancias negativas, si el resultado es menor a $0 \mathrm{~mm}$

b. Discrepancias positivas, si es mayor a Omm.

c. Discrepancias nulas, si el resultado es $0 \mathrm{~mm}$.

De acuerdo al análisis de estas discrepancias podemos determinar que tenemos:

Discrepancia nula: cuando el espacio disponible es igual al espacio requerido, es decir, el tamaño óseo es el necesario para albergar las piezas dentarias permanentes próximas en erupcionar, es decir las piezas 14, 15, 24, 25, $34,35,44,45$.

Discrepancia positiva: cuando el espacio disponible es mayor al espacio requerido; en este caso habrá diastemas o espacios entre los molares temporales, que ofrece espacio óseo sobrante para la erupción de los premolares, que podría presentar diastemas a futuro entre estas piezas.

Discrepancia negativa: cuando el espacio disponible es menor que el espacio requerido; no existirá espacio para los dientes permanentes no erupcionados, y se producirá a futuro apiñamiento dental, alteración de la relación molar y guía canina, pilares fundamentales de la oclusión.

\section{RESULTADOS}

Se evaluaron 68 modelos de estudio, dentro de los cuales, 38 pertenecen al género masculino y 30 al género femenino.

Dentro del grupo control, aproximadamente el 70\% de las arcadas superiores valoradas tuvieron una discrepancia positiva, que corresponde a 26 de los 38 modelos medidos; el 18\% una discrepancia nula, es decir 7 modelos de 38, mientras que solamente el $13 \%$ tuvo una discrepancia negativa, correspondiente a 5 modelos.

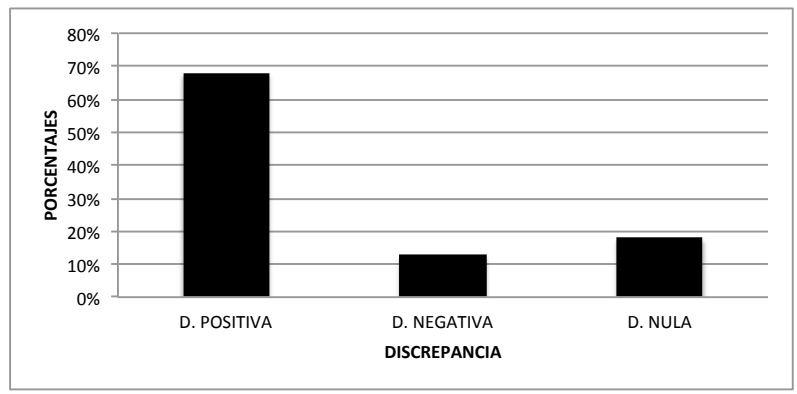

Figura $N^{\circ}$ 2. Porcentaje de discrepancia grupo I arco superior. 
Por otro lado, para las arcadas inferiores valoradas del grupo control, el 95\% de las mismas resultaron en discrepancia positiva, correspondiente a 36 modelos de estudio, mientras que el $5 \%$ fue negativo, solamente con 2 modelos. En este caso, no hubo discrepancia nula.

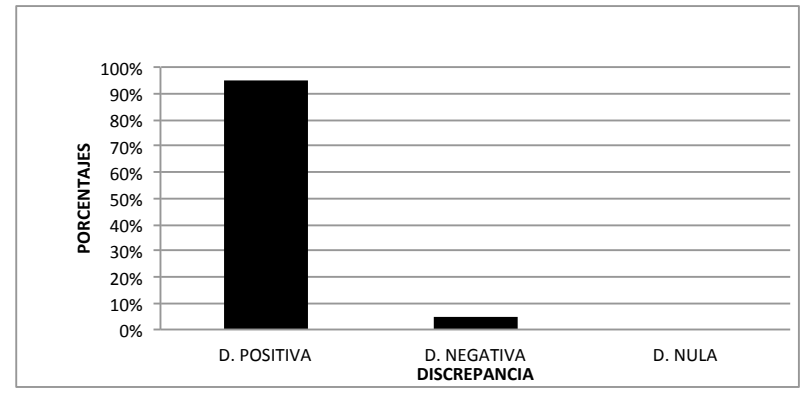

Figura № 3 . Porcentaje de discrepancia grupo I arco inferior.

En este caso podemos correlacionar que las discrepancias positivas son en mayor porcentaje; es decir, tenemos mayor espacio para la erupción de las piezas dentarias definitivas no erupcionadas, con la posibilidad de tener diastemas a futuro entre las mencionadas.

En cuanto al grupo II (ausencia de por lo menos un molar temporal en arco superior), se pudo observar que el $43 \%$ de la muestra obtuvo un resultado de discrepancia positiva, lo que corresponde a 13 modelos de estudio, mientras que el 50\% de la muestra, es decir 15 modelos, al ser medidos, resultaron con discrepancia negativa, y solamente el $6 \%$ obtuvo una discrepancia nula, correspondiente a 2 modelos.

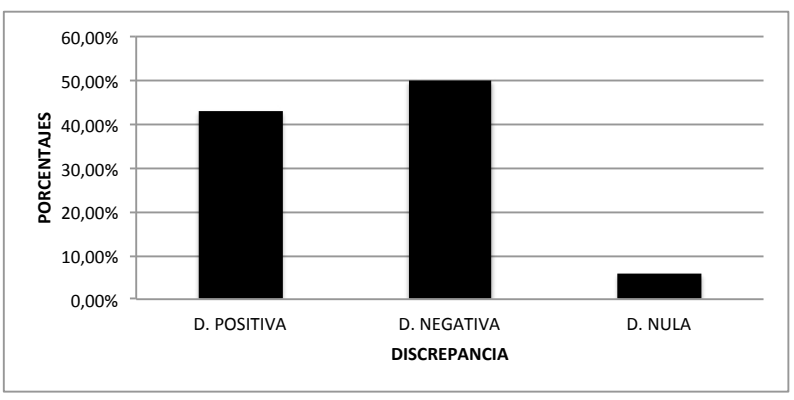

Figura $N^{\circ} 4$. Porcentaje de discrepancia grupo II arco superior.

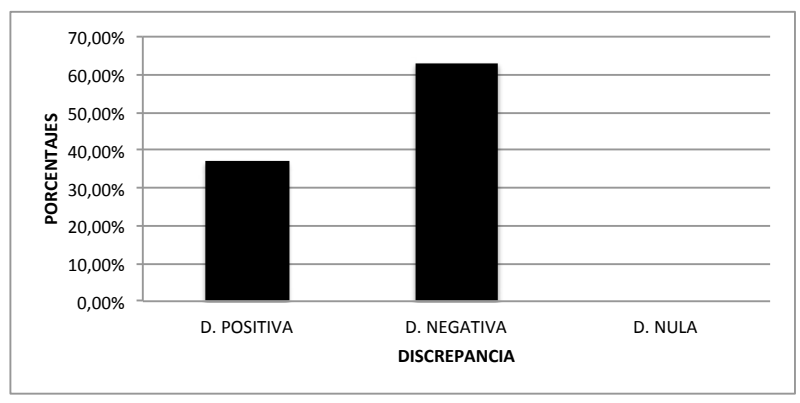

Figura $N^{\circ} 5$. Porcentaje de discrepancia grupo II arco inferior.

En el grupo II podemos observar claramente cómo la discrepancia negativa aumentó en gran porcentaje, debido a la pérdida de un molar temporal, lo que determinó que en estos niños, a futuro pueden presentar apiñamiento dental en sus arcadas dentarias, afectando su proceso de erupción normal, así como la guía canina y relación molar futura, que constituyen guías básicas para una correcta oclusión.

Mediante el programa SPSS, se encontró que, según la prueba T de igualdad de medias, Sig. $=0.444>0.05$ para el arco superior y Sig $=0.275>0.05$, para el arco inferior; por lo tanto, no es significativo, y no se puede afirmar que el tamaño del arco superior o del arco inferior, se encuentra influenciado por el sexo del paciente.

De la misma manera, mediante un análisis de la varianza, se pudo determinar que Sig. = 0. $265>0.05$, para el arco superior, y que Sig. $=0.784>0.05$; por lo tanto, no es significativo; entonces, el tamaño de los arcos dentales no se encuentra influenciado por la edad del paciente.

Por último, para poder determinar si la longitud del arco dentario es distinta con presencia de todas las piezas temporales o ausencia de por lo menos un molar temporal, se utilizó el programa Minitab, en el que se pudo determinar que Sig. $=0.002<0.05$ para el arco superior; entonces, los niños que presentan todos sus molares temporales, tienen una longitud mayor del arco superior que aquellos niños que han perdido por lo menos un molar temporal.

Para el arco inferior, Sig. $=0.000<0.05$, lo que indica que los niños que presentan todos sus molares temporales tienen una medida mayor del arco inferior que aquellos niños que han perdido por lo menos un molar temporal.

\section{DISCUSIÓN}

En el caso de los valores para las arcadas inferiores del grupo II, podemos observar que la discrepancia positiva se presenta en el 37\% de las muestras, correspondiente a 11 modelos de estudio, mientras que la discrepancia negativa en un 63\%, que representan 19 modelos. Aquí no se obtuvieron discrepancias nulas de ningún modelo.

\footnotetext{
| 56 | REVISTA EÍDOS, diciembre 2014
}

De acuerdo con los resultados obtenidos en el presente estudio, vale recalcar que el percentil utilizado para la predicción de espacios de premolares y caninos no erupcionados fue del 75\%; debido a que este valor representa una medida con un mayor margen de seguridad, 
una tendencia mayor de esta, estaría hacia el apiñamiento. (Vellini, F. 2002)

Por otro lado, según Flores, Bernabé y Col, tras realizar un estudio en 248 modelos de Trujillo-Perú, determinaron que para las mujeres es aplicable el percentil al 95\% en el arco superior, y al 65\% en el arco inferior, mientras que para hombres el 65\% es el adecuado para el arco inferior y por el contrario, ningún porcentaje se asemejaba a los valores estudiados del arco superior

Sin embargo, se debe tomar en cuenta que el método de Moyers fue realizado en pacientes caucásicos, lo que podría contraponerse con los resultados encontrados, debido a que nuestra población es mestiza, multiétnica, y lamentablemente, no existen parámetros establecidos para la población ecuatoriana.

Se debe tomar en cuenta que existen causas intrínsecas que influyen en las mediciones, como lo es la anatomía dental que como bien se sabe, es muy variable.

El método elaborado por Robert Moyers en 1973, para predecir los tamaños de los caninos y premolares no erupcionados, se sustenta en la buena relación que existe entre el tamaño de los incisivos permanentes inferiores y el de los caninos y premolares sin erupcionar. Por el contrario, reportó que los incisivos superiores muestran amplia variabilidad de tamaños, por lo que correlación no es óptima.

Es por esto que, en el estudio realizado, se han tomado los parámetros anteriores, es decir, las mediciones de los incisivos inferiores permanentes, mas no las mediciones de incisivos superiores.

\section{CONCLUSIONES}

a) Después de realizar el análisis estadístico, se puede concluir que, tanto para el caso de arcada superior y arcada inferior del grupo I o grupo control, la discrepancia positiva predomina.

b) En el caso del grupo II, la mitad de la muestra mantuvo un alto porcentaje de discrepancia negativa; sin embargo, la discrepancia positiva también estuvo muy marcada, con 13 de los 30 modelos.

c) En el grupo II, en la arcada superior se pudo observar un alto predominio de discrepancia negativa.

d) El hecho de que la discrepancia negativa sea más marcada en arcadas inferiores que superiores, del grupo II, nos da a entender que la incidencia de pérdidas tempranas de molares temporales es mayor en arco inferior que en arco superior.

e) No hay evidencia estadística, que indique que la medida de los arcos superior e inferior, se vea influenciada por el sexo del paciente.

f) No hay evidencia que indique que la medida de los arcos superior e inferior esté relacionada con la edad del paciente (7 a 9 años).

g) Se concluye que los pacientes que presentan en boca todas sus piezas molares temporales tienen, en promedio, un mayor tamaño tanto del arco superior como inferior, en comparación a los pacientes con ausencia de por lo menos uno de sus molares temporales.

h) De las dos conclusiones anteriores se deduce que la presencia de todas las piezas deciduas es un factor favorable para la futura erupción de las piezas permanentes, ya que estas van a tener una posición adecuada, dentro de la arcada dentaria.

i) Al contrario, la ausencia de por lo menos un molar temporal, produce una reducción de la longitud del arco dentario, por lo que hay una pérdida de espacio, con posibles alteraciones funcionales y estéticas en el futuro.

\section{REFERENCIAS BIBLIOGRÁFICAS}

Bordoni, Escobar. Castillo. (2010). Odontología Pediátrica: La salud bucal del niño y el adolescente en el mundo actual. Buenos Aires: Ed. Médica Panamericana.

Botero P. Pedroza A. \& Vélez N. (2007). Manual para la realización de historia clínica odontológica del escolar. Medellín:: Universidad Cooperativa de Colombia,

Cabello N. Mendoza V.M. Parés F.L. (2004). Valoración de la exactitud de predicción del tamaño dental mesiodistal de las tablas de probabilidad de Moyers y las ecuaciones de Tanaka-Johnston en una población mexicana. Revista ADM. LXI 5.

D’Escriván L. (2010). Ortodoncia en dentición mixta. Caracas: Ed. Amolca.

Dechkunakorn S. Chaiwat J. Sawaengkit P. (1990). Accuracy of Moyers' probability chart in a group of Thai subjects. J Dent Assoc Thai. 40(3),94-9.

Egoavil Lavado J. (2000). Aplicabilidad de los índices de Hixon-Oldfather y Moyers en la identificación del espacio requerido mandibular en la población peruana. (Tesis para optar el título de cirujano dentista). Perú. 
Feijoó García G. (2011). Cronología de la odontogénesis de los dientes permanentes en niños de la comunidad de Madrid: aplicación a la estimación de la edad dentaria. (Memoria para optar al grado de doctor). Universidad Complutense de Madrid, España.

Flores C. Bernabé E. Camus C. Carhuayo M. Major P. (2003). Prediction of mesiodistal canine and premolar tooth width in a sample of Peruvian adolescents Orthodontics \& Craniofacial Research. 6(3).

Flores J. (1995). Niveles de confianza del empleo de las tablas, de Moyers en 50 niños de 7 a 9 años de edad con oclusión normal, del distrito de Chorrillos. (Tesis para optar el título de cirujano dentista). Perú.

García A. (2009). Análisis de las características de dientes y arcadas primarias en población nomoclousiva. Recuperado de http://biblioteca.ucm.es/tesis/odo/ ucm-t28223.pdf.

Gutierrez Pulido, L. (2006). Validación de las tablas de probabilidad de Moyers en una población de LimaPerú, Lima-Perú.

Gutiérrez Pulido, L. (2006). Validación de las tablas de probabilidad de Moyers en una población de LimaPerú, Lima-Perú.

Hubertus, J.M. \& Van, W. \& Paul, W. S. (2002). Atlas de odontología pediátrica, Barcelona, España: Ed. Masson.

Moyers, R.E. (1992). Manual de ortodoncia. Buenos Aires: Ed. Médica Panamericana.

Negroni M, (2004). Microbiología estomatológica, fundamentos y guía práctica. Buenos Aires: Médica Panamericana.

Northway W. Wainright R. Demirjian A. (1984). Effects of premature loss of deciduous molars, The Angle Orthodontist. 54(4). 295-329.

Ortíz M. Godoy S. Farias M. Mata M. (2004-2005). Pérdida prematura de dientes temporales en pacientes de 5 a 8 años de edad asistidos en la Clínica de Odontopediatría de la Universidad Gran Mariscal de Ayacucho. Revista latinoamericana de ortodoncia y Odontopediatría. Recuperado de http://www. ortodoncia.ws.consultada.

Pinkham, J.R. (1996). Odontología Pediátrica. Pennsylvania: Ed. W.B Saunders

Ramírez M. Perona G. (2011). Enfermedades periodontales que afectan al niño y al adolescente, Odontol Ped, 10(1). 39-50.

Rosenfeld S. (2008). Estudio epidemiológico de maloclusiones en una población de 634 de entre 4 a 5 y 8 a 9 años de edad, de diversos colegios del área metropolitana de Quito. (tesis para obtener el título de odontólogo) USFQ. Quito, Ecuador.

Salas M. Aguilar F. \& col. Prevalencia de maloclusiones en adolscentes de 15 años del Gran Are Metropolitana. Costa Rica: Análisis de modelos. Recuperado de http://www.cienciasodontologicas.com/ odontohablando.html
Sandoval P. (2002). Manual de ortodoncia interceptiva: evolución de la dentición, capítulo II. Recuperado de http://www.med.ufro.cl/Recursos/ortodoncia/ images/Evolucion\%20Denticion.pdf.

Sonahita A. Dharma RM. (2012). Applicability of two methods of mixed dentition analysis in a contemporary Indian population sample. Eur J Paediatr Dent. 13 (1), 2934

Tabla de clasificación de la dentición permanente de Nolla (2012). Recuperado de http//:odonto42012.files. wordpress.com/2012/06/trabajo-osp-31.doc.

Tanaka M. (1974). The prediction of the size of unerupted canines and premolars in a contemporary osthodontic population. JADA. 68. 798-801.

Tootla R. Fayle S.A. (2003). Comparison of two methods of space prediction in the mixed dentition. Pediatr Dent. 25(4),350-356.

Uribe Restrepo G.A. (2004). Fundamentos de odontología, ortodoncia: Teoría y clínica. Medellín: Ed CIB.

Velásquez D. (2011). Análisis de Moyers y Tanaka y Jonhston, para la predicción del tamaño mesiodistal de caninos y premolares. (tesis para obtener el título de cirujanodentista) Lima, Perú.

Vellini, F. (2002). Ortodoncia diagnostico y planificación clínica. Sao Paulo: Artes Médicas.

58 | REVISTA EíDOS, diciembre 2014 